\title{
Probiotics for the Prevention of Ventilator-Associated Pneumonia: A Meta-Analysis of Randomized Controlled Trials
}

\author{
Minmin Su, Ying Jia, Yan Li, Dianyou Zhou, and Jinsheng Jia
}

\begin{abstract}
BACKGROUND: Ventilator-associated pneumonia (VAP) is a common and serious complication of mechanical ventilation. We conducted a meta-analysis of published randomized controlled trials to evaluate the efficacy and safety of probiotics for VAP prevention in patients who received mechanical ventilation. METHODS: We searched a number of medical literature databases to identify randomized controlled trials that compared probiotics with controls for VAP prevention. The results were expressed as odds ratios (OR) or mean differences with accompanying $95 \%$ CIs. Study-level data were pooled by using a random-effects model. Data syntheses were accomplished by using statistical software. RESULTS: Fourteen studies that involved 1,975 subjects met our inclusion criteria. Probiotic administration was associated with a reduction in VAP incidence among all 13 studies included in the meta-analysis $(\mathrm{OR} 0.62,95 \%$ CI $0.45-0.85 ; P=.003$; $\left.I^{2}=43 \%\right)$ but not among the 6 double-blinded studies (OR 0.72, 95\% CI 0.44-1.19; $P=.20 ; I^{2}=$ $55 \%$ ). We found a shorter duration of antibiotic use for VAP (mean difference $-1.44,95 \%$ CI -2.88 to $-0.01 ; P=.048, I^{2}=30 \%$ ) in the probiotics group than in the control group, and the finding comes from just 2 studies. No statistically significant differences were found between the groups in terms of ICU mortality (OR $0.95,95 \%$ CI $\left.0.67-1.34 ; P=.77 ; I^{2}=0 \%\right)$, ICU stay (mean difference $-0.77,95 \% \mathrm{CI}-2.58$ to $1.04 ; P=.40 ; I^{2}=43 \%$ ), duration of mechanical ventilation (mean difference $-0.91,95 \% \mathrm{CI}-2.20$ to $0.38 ; P=.17 ; \mathrm{I}^{2}=25 \%$ ), or occurrence of diarrhea (OR $0.72,95 \%$ CI $0.45-1.15 ; P=.17 ; I^{2}=41 \%$ ). CONCLUSIONS: The meta-analysis results indicated that the administration of probiotics significantly reduced the incidence of VAP. Furthermore, our findings need to be verified in large-scale, well-designed, randomized, multicenter trials. Key words: ventilator-associated pneumonia; critical care; probiotics; prevent; randomized controlled trial; meta-analysis. [Respir Care 2020;65(5):673-685. (C) 2020 Daedalus Enterprises]
\end{abstract}

\section{Introduction}

Ventilator-associated pneumonia (VAP) is defined as an infectious inflammatory reaction of the lung parenchyma that occurs after mechanical ventilation for $>48 \mathrm{~h}$. VAP is a common and severe complication in mechanically ventilated patients and can lead to a prolonged hospital stay, increased medical costs, and a higher mortality risk. ${ }^{1,2}$ The

\footnotetext{
Ms Su and Ms Jia are affiliated with the College of Pharmacy, Shanxi Medical University, Taiyuan, Shanxi, China. Mr Jia, Ms Li, and Ms Zhou are affiliated with the Department of Pharmacy, Shanxi Medical University Affiliated Jincheng General Hospital, Jincheng, Shanxi, China.

The authors have no conflicts of interest to declare.

Supplementary material related to this paper is available at http://www. rcjournal.com.
}

incidence of VAP in the United States is $\sim 4.4$ cases per 1,000 mechanical ventilation days, ${ }^{3}$ and it has become an important cause of death in patients who receive mechanical ventilation. The pathogenesis of VAP is mainly due to bacterial colonization of the upper respiratory tract and inhalation of contaminated secretions into the lower respiratory tract. ${ }^{4}$ Although antibiotics can effectively reduce the VAP incidence by eliminating pathogenic bacteria, ${ }^{4}$ antibiotic abuse can lead to an increase in drug resistance. Consequently, finding new safe and effective preventive measures is important. ${ }^{5}$

Correspondence: Jinsheng Jia MS, Department of Pharmacy, Shanxi Medical University Affiliated Jincheng General Hospital, Kaifa District, Jincheng City, ShanxiProv, China 048000.E-mail: 1329019376@qq.com.

DOI: 10.4187/respcare.07097 
Recently, the intestinal microflora has attracted great attention from researchers. Results of studies indicate that intestinal microecological imbalances are associated with the development of obesity, diabetes, and inflammatory diseases. ${ }^{6-8}$ Probiotics are defined as active microorganisms that can produce positive effects in the host when administered at the appropriate dosage. ${ }^{6}$ They can selectively stimulate the growth of some bacteria and improve the microecological balance of the host. ${ }^{9}$ The positive effects of probiotics on VAP may include (1) strengthening the gut barrier function, (2) reducing the overgrowth of potential pathogens, and (3) stimulating immune responses..$^{10,11}$

However, whether probiotics can effectively prevent VAP remains controversial. Currently, results of 6 randomized controlled trials (RCTs) indicate that the administration of probiotics has a positive effect on reducing VAP incidence. ${ }^{4,12-16}$ Results of 8 other RCTs show that probiotics had no significant effect on the prevention of VAP. ${ }^{17-20,22-25}$ In 2017, Weng et $\mathrm{al}^{26}$ conducted a meta-analysis and found that probiotics reduced the incidence of VAP in mechanically ventilated subjects. There may be great population heterogeneity in the meta-analysis by Weng et $\mathrm{al}^{26}$ because it included two articles that involved pediatric and neonatal subjects. In the 2 years since the meta-analysis by Weng et al, ${ }^{26} 3 \mathrm{RCTs}$ have been published. ${ }^{15,17,18}$ To elucidate the latest and most convincing evidence, we carried out a meta-analysis of the published RCTs to evaluate the effects of probiotics on VAP prevention in subjects on mechanical ventilation.

\section{Methods}

\section{Search Strategy}

Two of us (MS, YJ) systematically retrieved studies from PubMed, EMBASE, and Cochrane databases (each database was searched on February 28, 2019). The search phrases used included "probiotics" or "probiotic" or "synbiotics" or "synbiotic" or "Lactobacillus" or "lactobacilli" or "Bifidobacterium" and "pneumonia, ventilator-associated" or "pneumonia, ventilator associated" or "ventilator-associated pneumonia" or "VAP" or "nosocomial pneumonia" or "hospital acquired pneumonia" or "respiratory infection" or "critically ill" and "randomized, controlled trial" or "randomized" or "placebo." Our search strategy for PubMed is provided in the supplementary materials (see the supplementary materials at http://www.rcjournal.com).

\section{Study Selection and Outcome Assessment}

Eligibility Criteria. We included studies that met the following criteria: (1) involved adults receiving mechanical ventilation, (2) compared probiotics with a placebo or standard therapy, (3) assessed VAP as the outcome, and

\section{QUICK LOOK}

\section{Current knowledge}

Ventilator-associated pneumonia (VAP) is a common complication in mechanically ventilated patients. Many studies have evaluated the role of probiotics in the prevention of VAP, but there is no clear conclusion.

\section{What this paper contributes to our knowledge}

Our meta-analysis indicated that probiotics can effectively reduce the incidence of VAP. Additional largescale and multi-center randomized controlled trials are needed to further verify the role of probiotics in the prevention of VAP.

(4) were RCTs (including multi-arm random clinical studies).

Exclusion Criteria. We excluded studies that met the following criteria: (1) included only interventions with prebiotics and without any additional probiotics, and (2) analyzed the same cohort as other studies (we selected the one with the largest sample size or the longest follow-up time).

Outcome Assessment. The primary outcome was VAP incidence, and the secondary outcomes were ICU mortality, length of ICU stay, duration of mechanical ventilation, antibiotic use for VAP, and diarrhea. All the studies were screened independently by MS and YJ. After removing duplicate results, we skimmed through the topics and abstracts to preliminarily determine the eligible studies. We then evaluated the full texts to determine the studies that were ultimately included in the meta-analysis. Disagreements were resolved by discussion or by a third reviewer (YL).

\section{Data Extraction}

MS and YJ independently extracted data by using a Microsoft Excel spreadsheet (XP Professional Edition, Microsoft, Redmond, Washington); the extracted data included the study characteristics (the first author, publication year, design, duration, participants, interventions, definition of VAP) and the outcomes. Missing means and SDs were estimated by the methods of Luo et $\mathrm{al}^{19}$ and Wan et al, ${ }^{20}$ respectively. For multiple treatment arm studies, we pooled the data by using the methods described in the Cochrane Handbook. ${ }^{21}$ 


\section{PROBIOTICS FOR VAP PREVENTION}

\section{Quality Assessment}

The bias of the studies was assessed as described in the Cochrane Handbook ${ }^{21}$ and included characteristics such as random sequence generation, allocation concealment, blinding of patients, blinding of outcome assessments, completeness of outcome data, selective reporting, and other biases. Differences were resolved by discussion or by a third reviewer (YL).

\section{Data Pooling and Analysis}

We calculated the odds ratios (OR) with $95 \%$ CIs for binary variables and mean differences with $95 \%$ CIs for continuous variables. Heterogeneity among the studies was evaluated by the $\mathrm{I}^{2}$ statistics. Studies with $\mathrm{I}^{2}>50 \%$ were considered to have significant heterogeneity. The study-level data were pooled by using a random-effects model. Data analysis and synthesis were accomplished by using RevMan 5.3 (Cochrane Collaboration, Oxford, United Kingdom) and Stata 14.0 (Stata, College Station, Texas). Publication bias was also estimated by using a contour-enhanced funnel plot. We performed subgroup analyses for the different species of probiotics, diagnostic criteria for VAP, types of trial design. and pathogens. Sensitivity analyses were conducted by excluding studies with a high risk of bias and inexplicit diagnostic criteria to evaluate the stability of the primary outcome. In addition, a sensitivity analysis was performed to identify the source of heterogeneity among the studies.

\section{Results}

\section{Included Studies}

We retrieved a total of 413 articles from the above databases. A total of 283 articles remained after deleting duplicate records; 262 were excluded based on their titles and abstracts. After reading the full texts, 14 RCTs were included in the analysis. The study screening process is shown in Figure 1.

\section{Characteristics of the Included Studies}

The characteristics of all of the RCTs included in this meta-analysis are shown in Table $1 .^{4,12-18,22-25,27,28}$ Most of the included studies were published within the past 10 years. The sample sizes ranged from 35 to 300 (median, 150). Thirteen studies reported the VAP incidence, 6 reported ICU mortality, 10 reported ICU stays, 8 reported the duration of mechanical ventilation, 2 reported antibiotic use for VAP, and 6 reported the occurrence of diarrhea. Five studies used a single probiotic (Lactobacillus rhamnosus, ${ }^{14,23}$ Lactobacillus plantarum $299,{ }^{17,25}$ or Lactobacillus

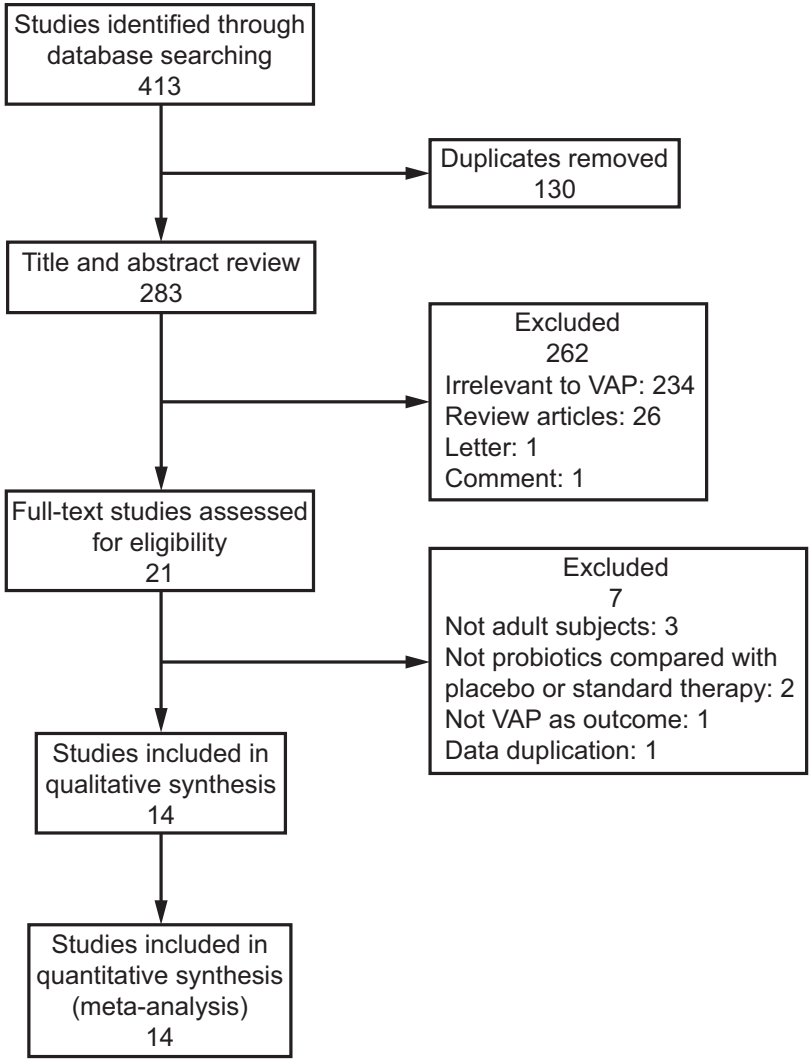

Fig. 1. Flow chart.

case $\left.^{26}\right), 5$ studies used multiple probiotics, ${ }^{4,15,18,22,28}$ and 4 studies used a synbiotic formula (Synbiotic 2000Forte Medipharm, Kågeröd, Sweden and Des Moines, IA). ${ }^{12,13,16,24}$ The studies included 7 double-blind trials, ${ }^{12-14,18,22-24} 3$ single-blind trials, ${ }^{15,16,28}$ and 4 open trials. ${ }^{4,17,25,27}$ With regard to the diagnostic criteria for VAP, 8 studies required microbiologic confirmation, ${ }^{13,17,18,22,23,25,27,28} 4$ studies used only clinical criteria, ${ }^{4,12,14,24}$ and 2 studies did not provide specific diagnostic criteria. ${ }^{15,16}$ The outcome data extracted from the RCTs included in the meta-analysis are shown in Table 2 .

\section{Risk of Bias in the Included Studies}

The risk of bias in the included studies is shown in Figures 2 and 3. Five studies were considered to have a high risk bias ${ }^{4,14,17,25,27}$ for the following reasons: the trials were open studies and did not perform blinding of participants and personnel, ${ }^{4,17,25,27}$ there were no blind evaluations of the outcome, ${ }^{25,27}$ and the reasons for the lack of data among the groups were not similar. ${ }^{4,14}$ In addition, 7 studies $^{14-16,22-24,28}$ reported random sequence generation, 6 studies $^{15,16,22-24,28}$ reported allocation concealment, and all of the studies were estimated to have a low risk of reporting bias. 


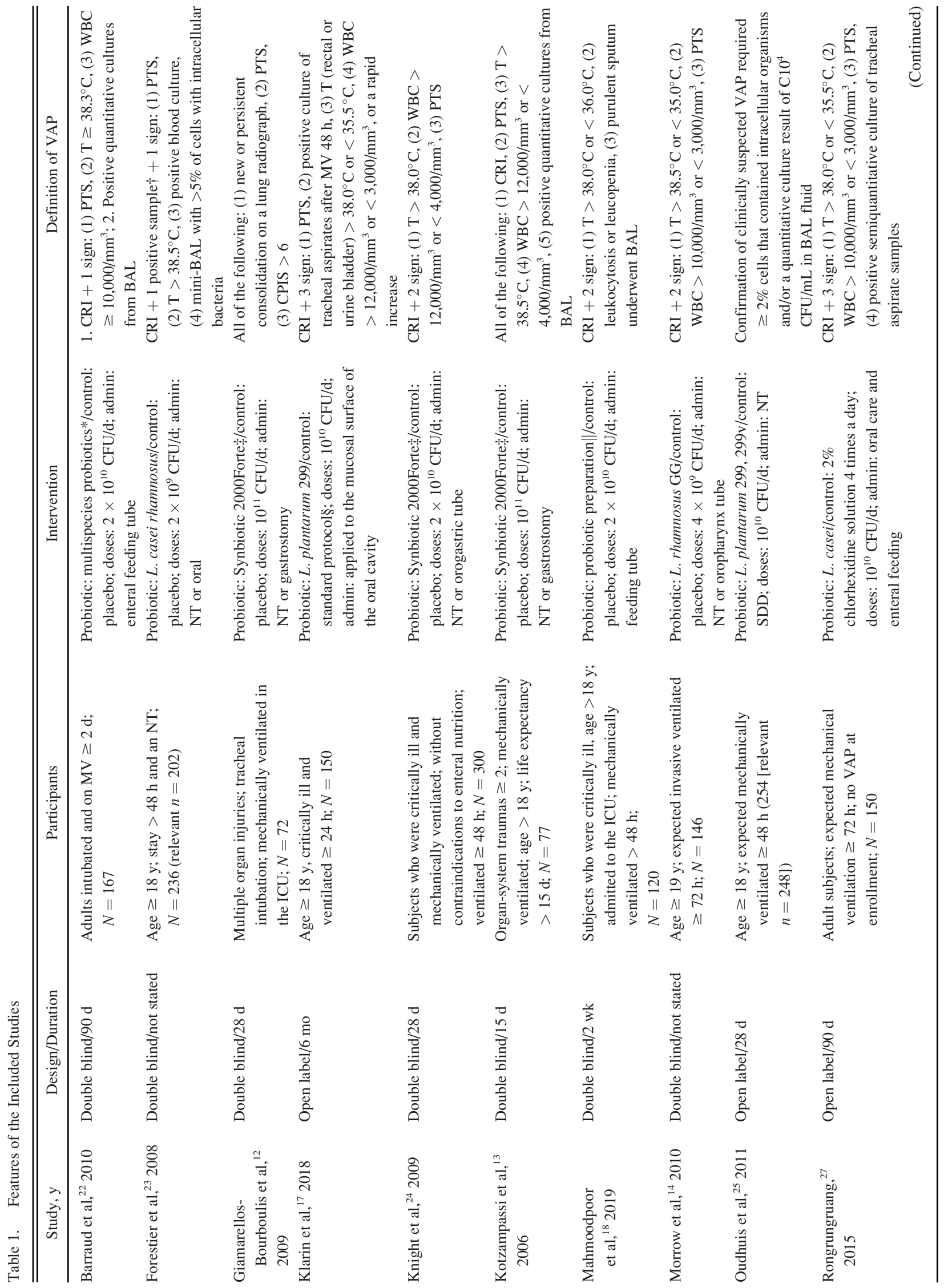


PROBIOTICS FOR VAP PREVENTION

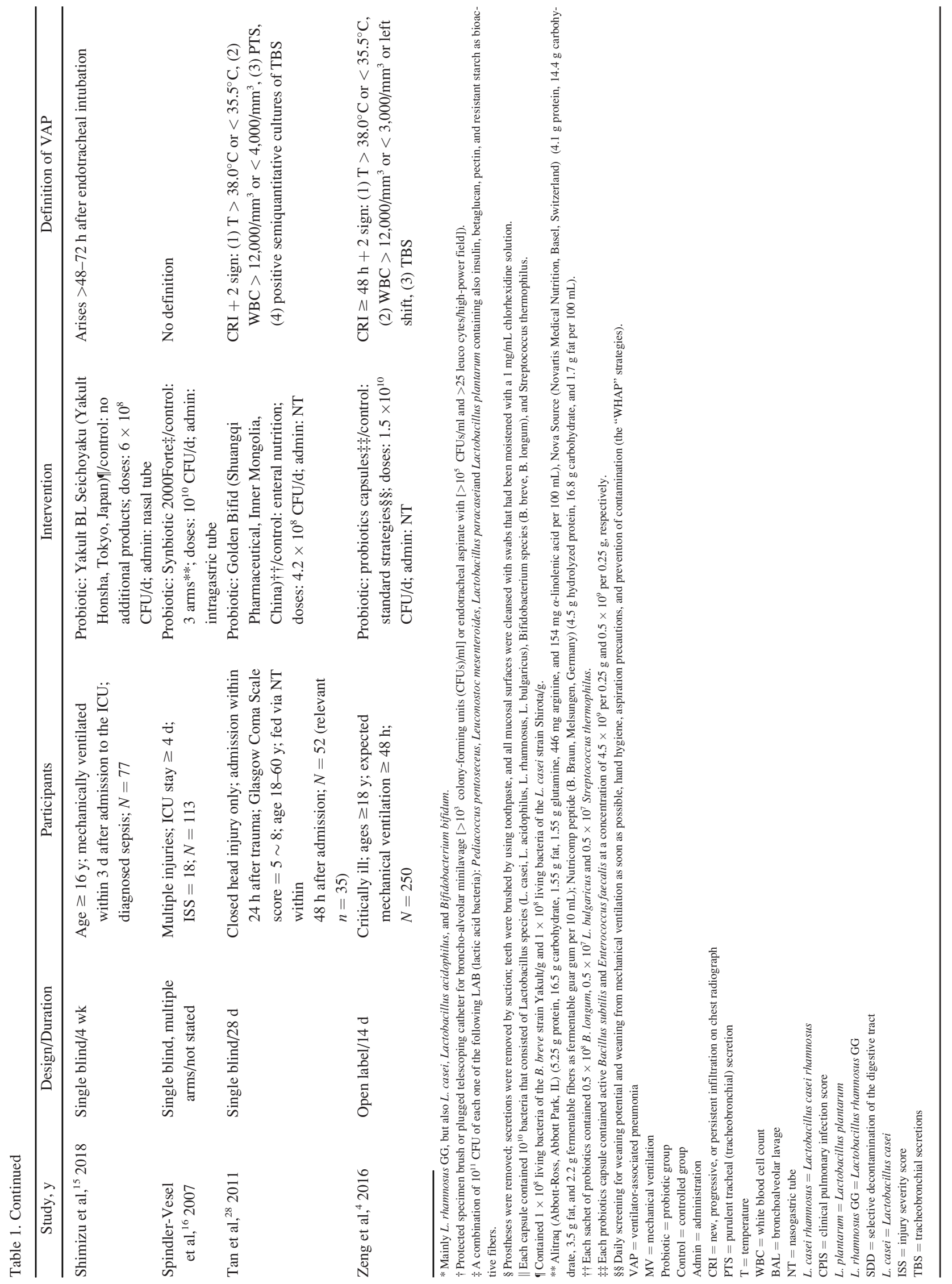




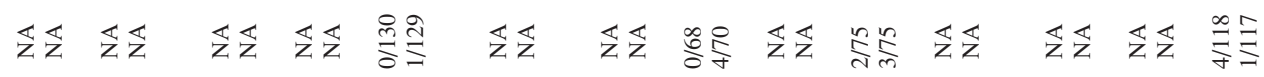

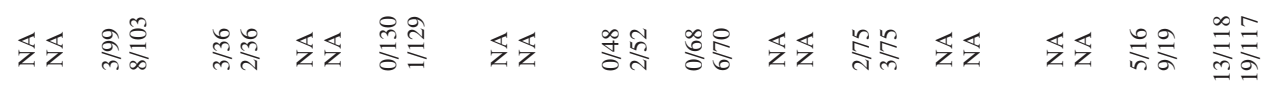

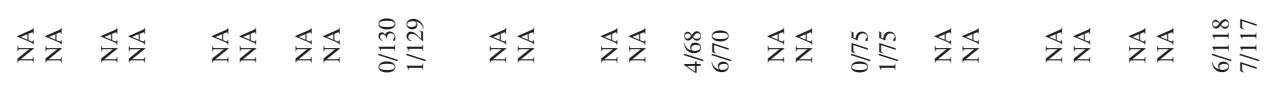

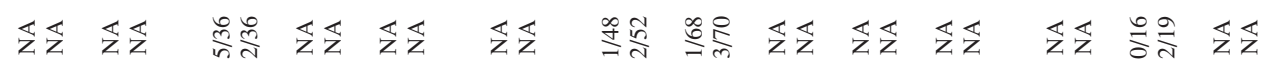

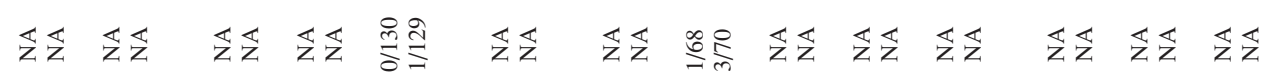

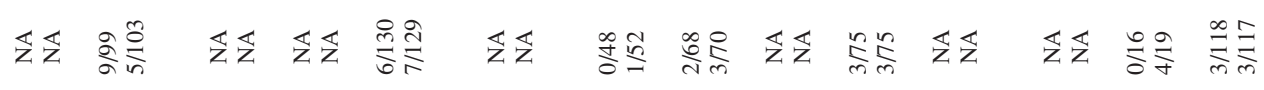

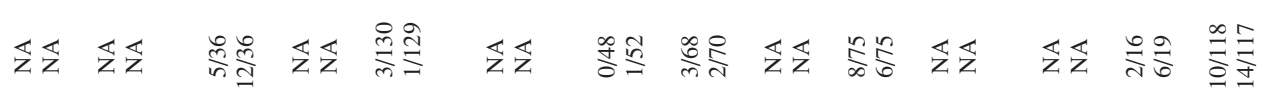

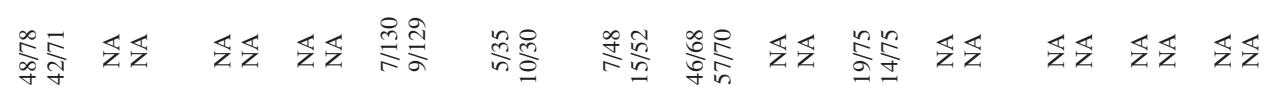

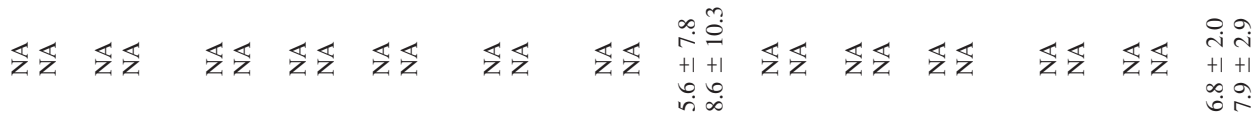

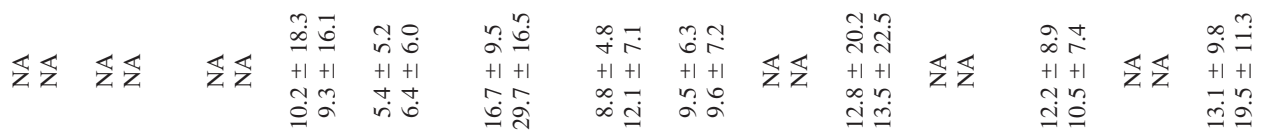

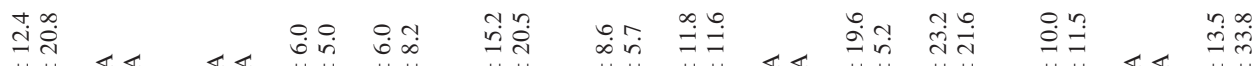

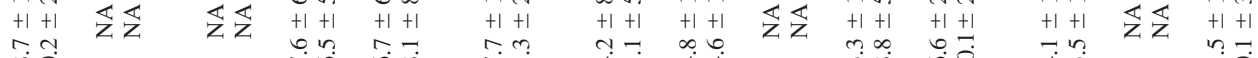



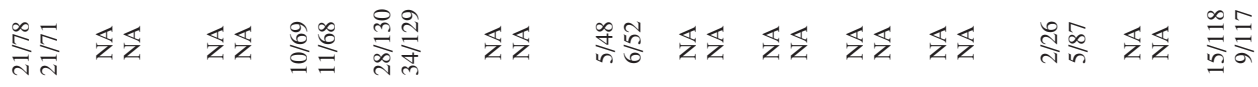

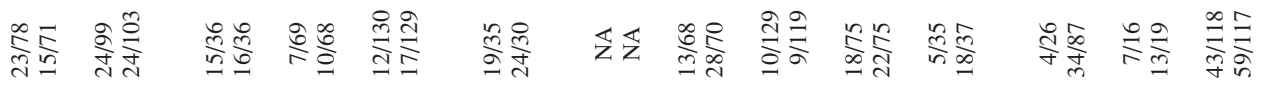




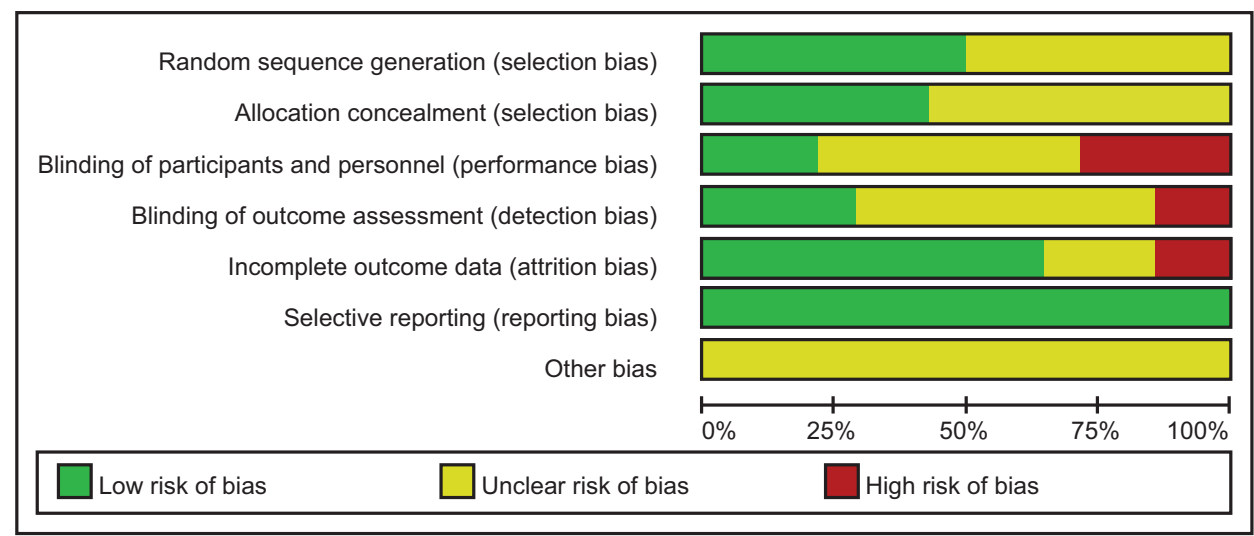

Fig. 2. Judgments about each risk of bias item.

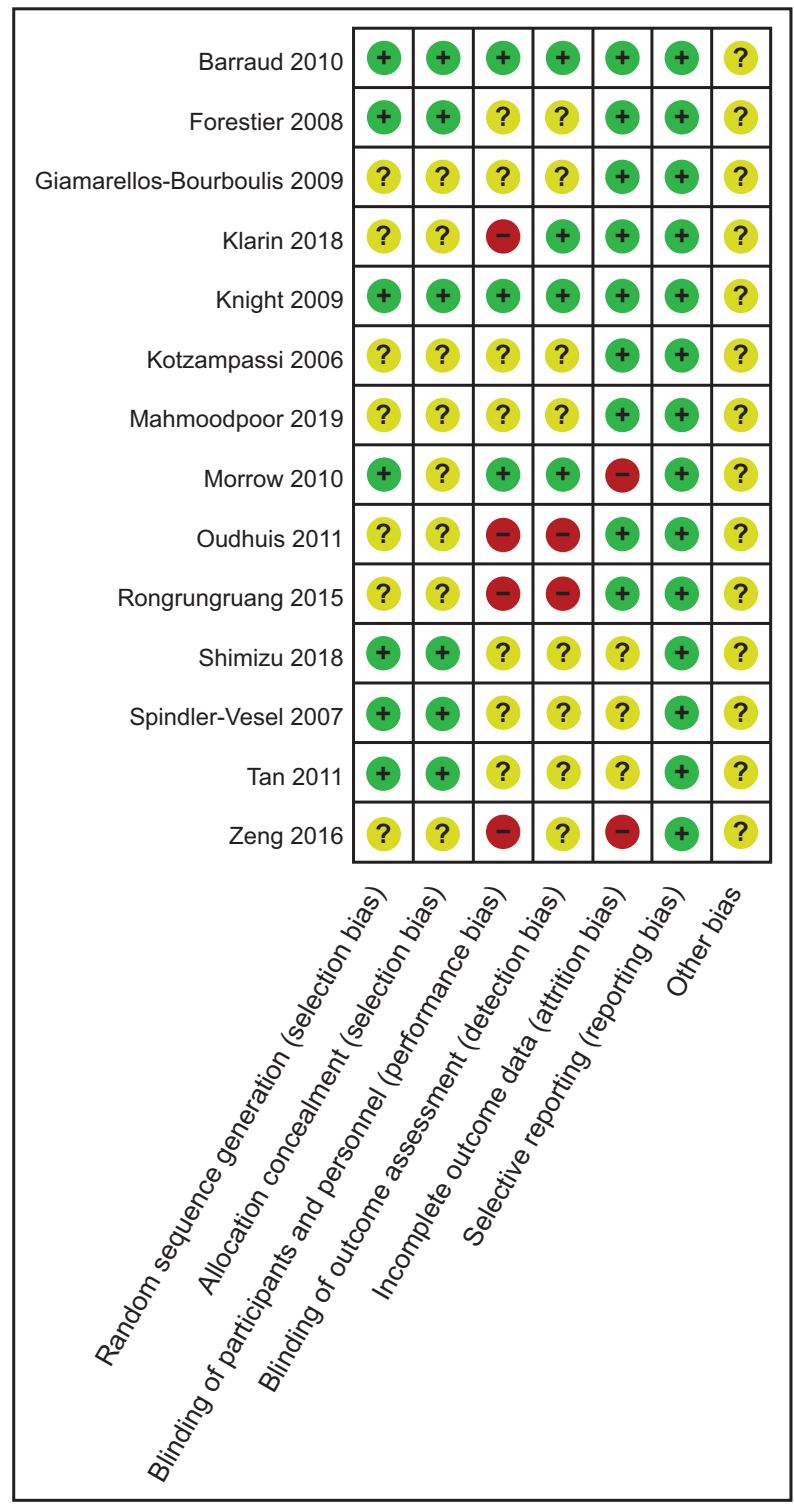

Fig. 3. Judgments about each risk of bias item for each included study.

\section{Outcomes}

We performed our meta-analysis based on a per-protocol analysis and intention-to-treat analysis, and the results were consistent. The intention-to-treat analysis is provided in the supplementary materials (see the supplementary materials at http://www.rcjournal.com).

Primary Outcome: VAP Incidence. Thirteen RCTs $(1,875$ subjects) reported a VAP incidence. The analysis showed that a VAP incidence in the probiotics group was significantly lower than that in the control group (OR 0.62, 95\% CI $0.45-0.85 ; P=.003 ; \mathrm{I}^{2}=43 \%$ ) (Fig. 4). After removing the studies with a high risk of bias ${ }^{4,14,17,25,27}$ and inexplicit diagnostic criteria, ${ }^{15,16}$ the results remained statistically significant.

Secondary Outcomes. The secondary outcomes are summarized in Table 3. No statistically significant differences were found between the groups in terms of ICU mortality (Fig. 5), ICU stay (Figs. 6 and 7), duration of mechanical ventilation (Figs. 8 and 9), or occurrence of diarrhea (Fig. 10). We found a shorter duration of antibiotic use for VAP in the probiotics group than in the control group (Fig. 11).

\section{Subgroup Analyses}

The results of subgroup analysis for the primary outcome are outlined in Table 4; further details are provided in the supplementary materials (see the supplementary materials at http://www.rcjournal.com).

\section{Publication Bias}

A contour-enhanced funnel plot (Fig. 12) revealed apparent asymmetry that suggested the presence of a potential publication bias. 


\section{PROBIOTICS FOR VAP PREVENTION}

\begin{tabular}{|c|c|c|c|c|c|c|c|c|c|c|}
\hline \multirow[b]{2}{*}{ Study or Subgroup } & \multicolumn{2}{|c|}{ Experimental } & \multicolumn{2}{|c|}{ Control } & \multicolumn{3}{|c|}{ Odds Ratio } & \multirow{2}{*}{\multicolumn{2}{|c|}{$\begin{array}{c}\text { Odds Ratio } \\
\mathrm{M}-\mathrm{H}, \text { Random, } 95 \% \mathrm{Cl}\end{array}$}} & \\
\hline & Events & Total & Events & Total & Weight, \% & $\mathrm{M}-\mathrm{H}$, Random, $95 \% \mathrm{Cl}$ & & & & \\
\hline Barraud 2010 & 23 & 78 & 15 & 71 & 9.1 & $1.56(0.74-3.30)$ & & & & \\
\hline Forestier 2008 & 24 & 99 & 24 & 103 & 10.5 & $1.05(0.55-2.01)$ & & & - & \\
\hline Giamarellos-Bourboulis 2009 & 15 & 36 & 16 & 36 & 7.1 & $0.89(0.35-2.27)$ & & & & \\
\hline Klarin 2018 & 7 & 69 & 10 & 68 & 6.3 & $0.65(0.23-1.83)$ & & & - & \\
\hline Knight 2009 & 12 & 130 & 17 & 129 & 8.7 & $0.67(0.31-1.47)$ & & & & \\
\hline Kotzampassi 2006 & 19 & 35 & 24 & 30 & 5.6 & $0.30(0.10-0.91)$ & & & & \\
\hline Morrow 2010 & 13 & 68 & 28 & 70 & 8.9 & $0.35(0.16-0.77)$ & & & & \\
\hline Oudhuis 2011 & 10 & 129 & 9 & 119 & 7.1 & $1.03(0.40-2.62)$ & & & & \\
\hline Rongrungruang 2015 & 18 & 75 & 22 & 75 & 9.4 & $0.76(0.37-1.57)$ & & & - & \\
\hline Shimizu 2018 & 5 & 35 & 18 & 37 & 5.4 & $0.18(0.06-0.55)$ & & & & \\
\hline Spindler-Vesel 2007 & 4 & 26 & 34 & 87 & 5.4 & $0.28(0.09-0.89)$ & & & & \\
\hline Tan 2011 & 7 & 16 & 13 & 19 & 4.1 & $0.36(0.09-1.43)$ & & & & \\
\hline Zeng 2016 & 43 & 118 & 59 & 117 & 12.4 & $0.56(0.33-0.95)$ & & & & \\
\hline Total $(95 \% \mathrm{Cl})$ & & 914 & & 961 & 100 & $0.62(0.45-0.85)$ & & & & \\
\hline Total events & 200 & & 289 & & & & & & & \\
\hline $\begin{array}{l}\text { Heterogeneity: } \mathrm{Tau}^{2}=0.14 ; \mathrm{Ch} \\
\text { Test for overall effect: } Z=2.99\end{array}$ & $\begin{array}{l}\mathrm{i}^{2}=21.22 \\
(P=.003\end{array}$ & $d f=1$ & $2(P=.05$ & $5) ; 1^{2}=$ & $43 \%$ & & 0.01 & $\begin{array}{l}0.1 \\
\text { ors probiotics }\end{array}$ & $\begin{array}{lr}1 & 1 \\
& \text { Favors co }\end{array}$ & $\begin{array}{ll}10 & 100 \\
\text { ontrol }\end{array}$ \\
\hline
\end{tabular}

Fig. 4. A forest plot of ventilator-associated pneumonia (VAP) incidences.

Table 3. The Secondary Outcomes

\begin{tabular}{|c|c|c|c|c|}
\hline Outcome or Subgroup Title & Studies, no. & Subjects, $N$ & Statistical Method & Effect Size \\
\hline ICU mortality & 6 & 993 & OR (M-H, random, 95\% CI) & $0.95(0.67,1.34)$ \\
\hline Length of ICU stay, $d$ & 10 & 1,418 & Mean difference (IV, random, 95\% CI) & $-1.29(-4.74,2.15)$ \\
\hline Removed studies that led to high heterogeneity & 7 & 1,103 & Mean difference (IV, random, 95\% CI) & $-0.77(-2.58,1.04)$ \\
\hline Duration of mechanical ventilation, $d$ & 8 & 1,197 & Mean difference (IV, random, 95\% CI) & $-2.37(-4.67,-0.08)$ \\
\hline Removed studies that led to high heterogeneity & 6 & 897 & Mean difference (IV, random, 95\% CI) & $-0.91(-2.20,0.38)$ \\
\hline Antibiotic use for VAP, $\mathrm{d}$ & 2 & 373 & Mean difference (IV, random, 95\% CI) & $-1.44(-2.88,-0.01)$ \\
\hline Occurrence of diarrhea & 6 & 861 & OR $(\mathrm{M}-\mathrm{H}$, random, $95 \% \mathrm{CI})$ & $0.72(0.45,1.15)$ \\
\hline $\begin{array}{l}\mathrm{OR}=\text { odds ratio } \\
\mathrm{M}-\mathrm{H}=\text { Mantel-Haenszel } \\
\mathrm{IV}=\text { inverse variance } \\
\mathrm{VAP}=\text { ventilator-associated pneumonia }\end{array}$ & & & & \\
\hline
\end{tabular}

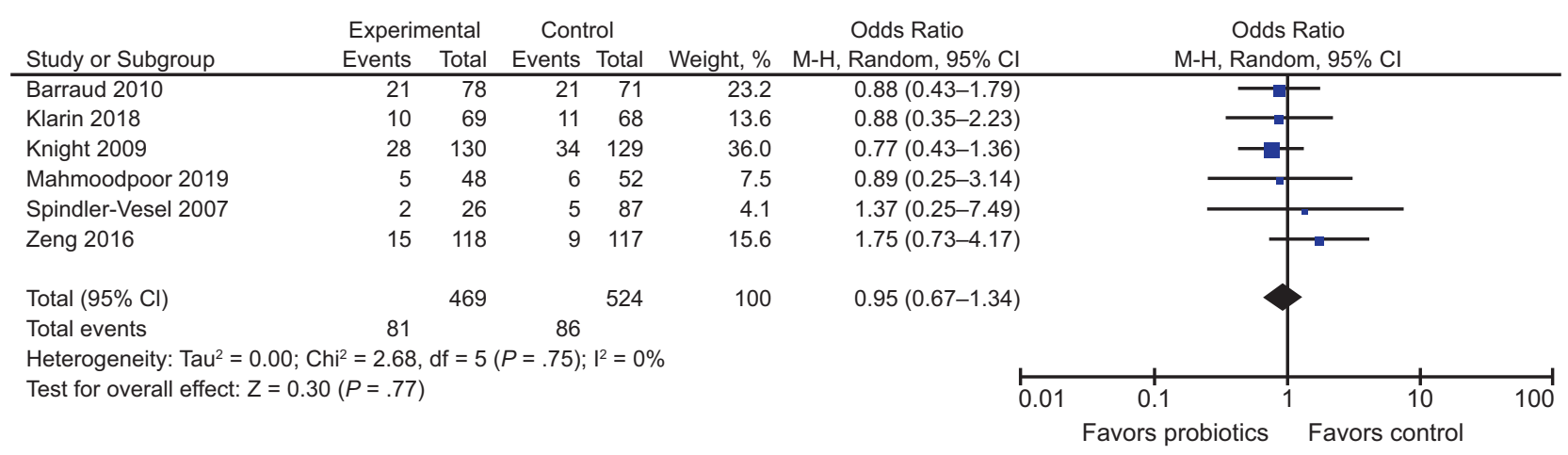

Fig. 5. A forest plot of ICU mortality.

\section{Discussion}

Our meta-analysis demonstrated that probiotics significantly decreased the incidence of VAP. No appreciable effects were conferred by probiotics on ICU mortality, length of ICU stay, duration of mechanical ventilation, or occurrence of diarrhea. We found a reduction in the use of antibiotics for VAP among the subjects treated with probiotics, but an insufficient sample limited the strength of this result. Although no adverse effects related to probiotics were found in the included studies, it is vital to conduct safety monitoring in future clinical trials. 


\section{PROBIOTICS FOR VAP PREVENTION}

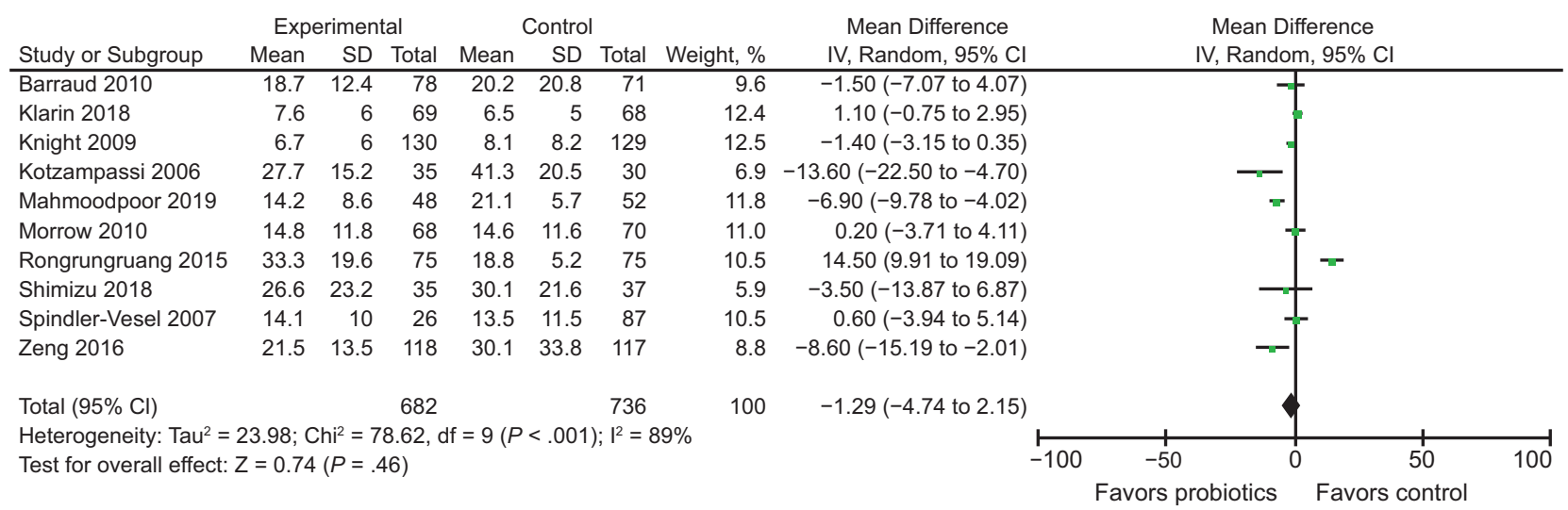

Fig. 6. A forest plot of length of ICU stay (days).

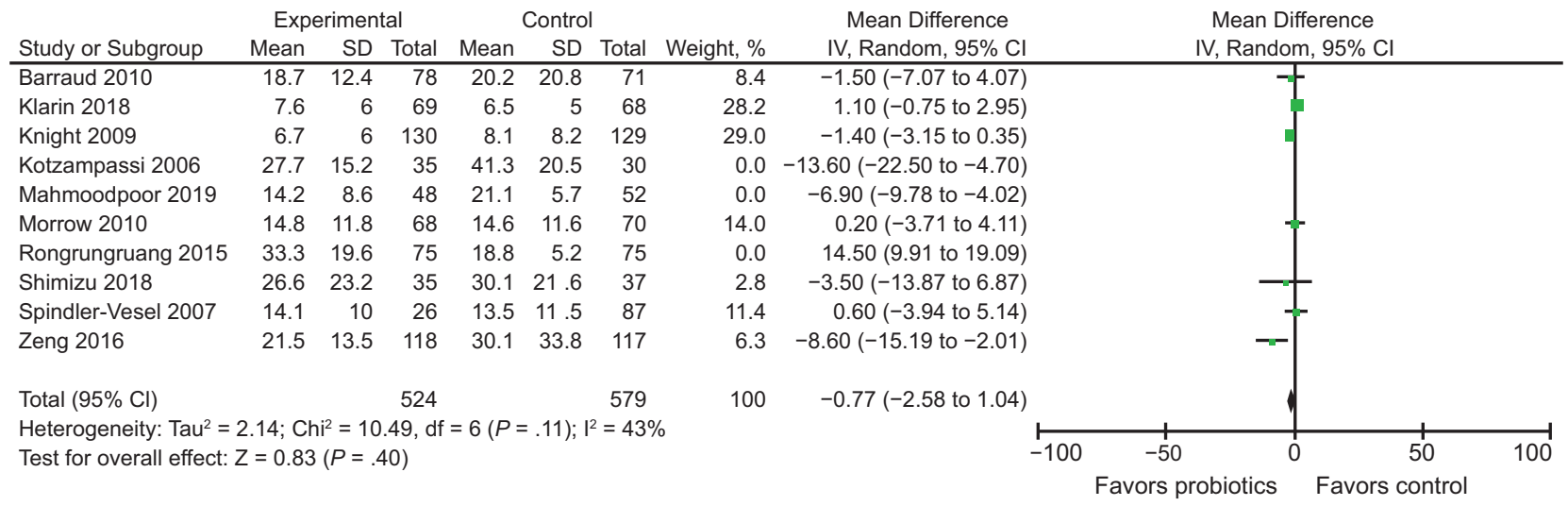

Fig. 7. A forest plot of the sensitivity analysis for length of ICU stay (days).

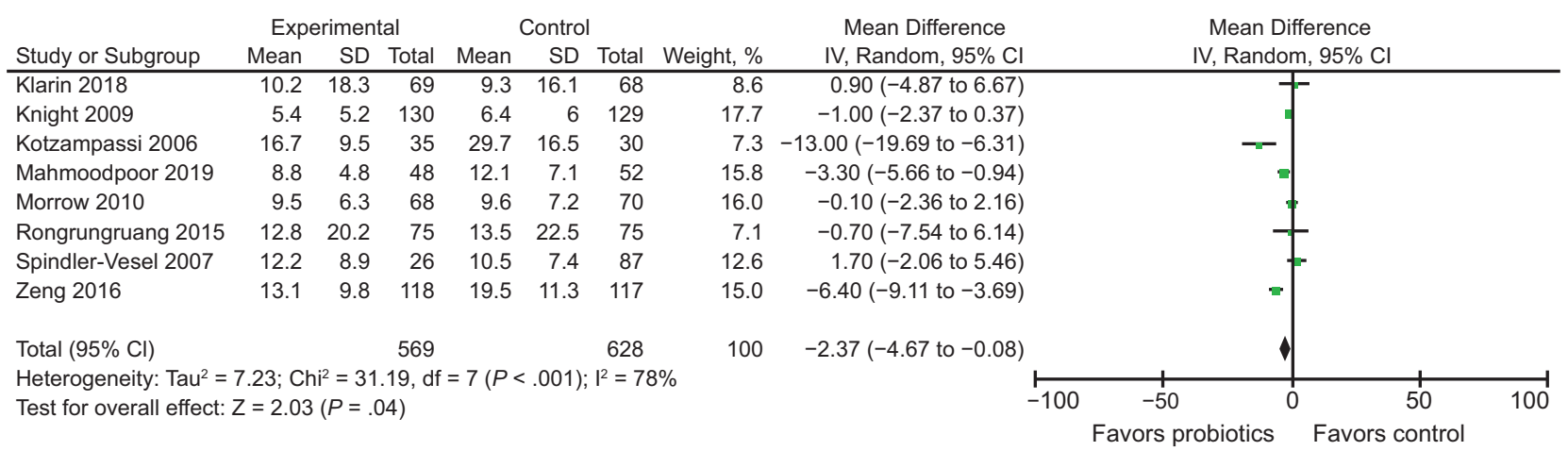

Fig. 8. A forest plot of duration of mechanical ventilation (days).

Our conclusions were consistent with 3 previously published meta-analyses (Weng et al, ${ }^{26}$ Bo et al, ${ }^{29}$ and Siempos et $\left.\mathrm{al}^{30}\right)$. By including more recent studies, our meta-analysis further confirmed these findings. With regard to the metaanalysis by Weng et $\mathrm{al}^{26}$ we did not think that two of the studies $^{31,32}$ were appropriate for inclusion because the participants in Banupriya et $\mathrm{al}^{31}$ and $\mathrm{Li}$ et $\mathrm{al}^{32}$ were children and neonate, respectively. The intestinal microenvironment of children is significantly different from that of adults.
This clinical heterogeneity may have caused bias in the outcome. We excluded these 2 studies and included 3 newly published RCTs (Mahmoodpoor et al, ${ }^{18}$ Klarin et al, ${ }^{17}$ and Shimizu et $\mathrm{al}^{15}$ ) and one other RCT (Kotzampassi et $\mathrm{al}^{13}$ ), which made our results more convincing. The study by Kotzampassi et $\mathrm{al}^{13}$ was also included in the studies of Bo et $\mathrm{al}^{29}$ and Siempos et al. ${ }^{30}$ The meta-analysis of $\mathrm{Wang}^{33}$ suggested that probiotics had no effect on VAP prevention; however, differences in the probiotic strains and VAP 


\section{PROBIOTICS FOR VAP PREVENTION}

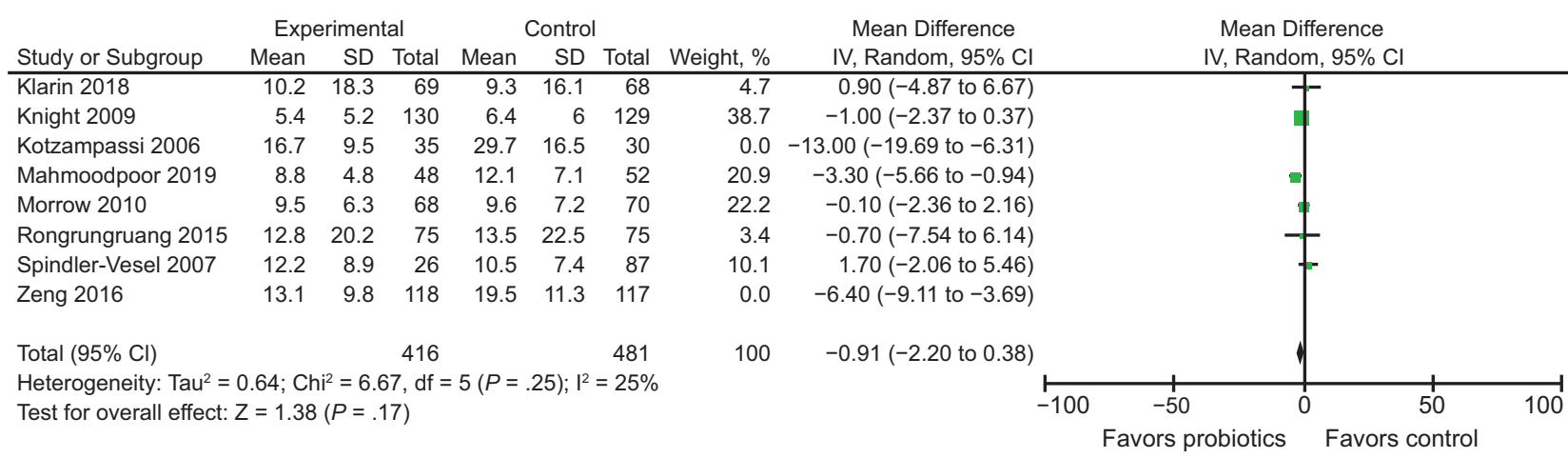

Fig. 9. A forest plot of the sensitivity analysis for duration of mechanical ventilation.

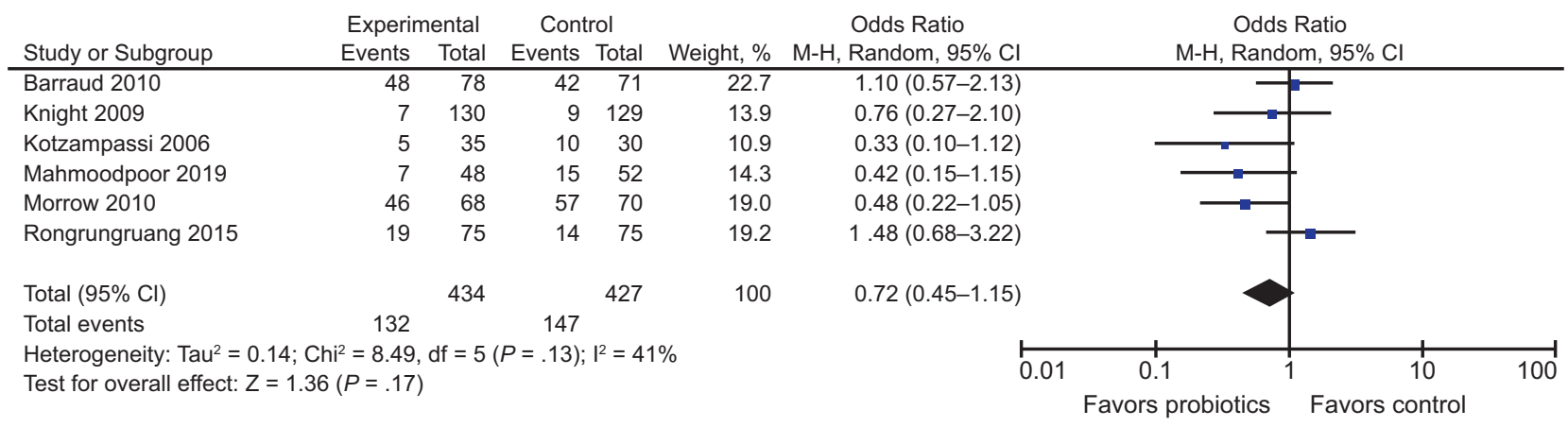

Fig. 10. A forest plot of occurrence of diarrhea.

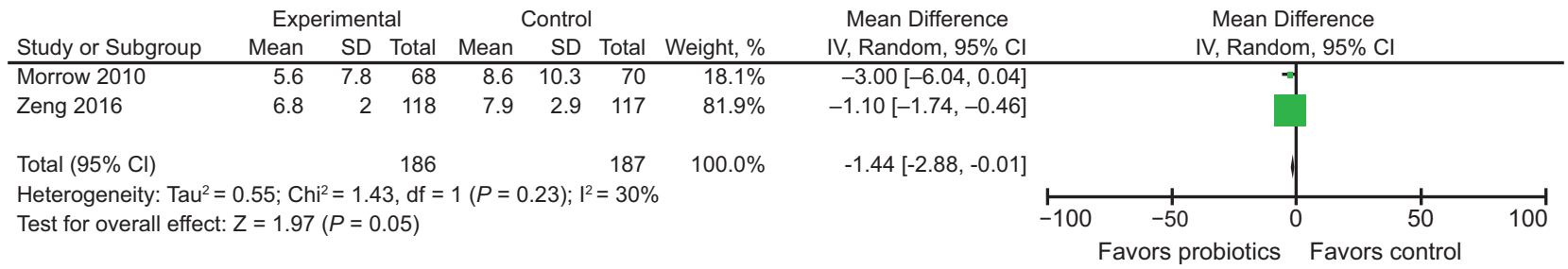

Fig. 11. A forest plot of antibiotic use for ventilator-associated pneumonia (VAP) (days).

diagnostic criteria may be the reason for the inconsistent results. An insufficient sample size greatly weakened their conclusions.

To date, measures to prevent VAP mainly include subglottic secretion drainage, the use of oral antiseptics, and a shortened duration of mechanical ventilation, but only a limited number of positive results have been achieved. ${ }^{10}$ Analysis of a substantial number of findings suggested that probiotics were a promising option for VAP prevention. For instance, probiotics stimulate immune responses by increasing phagocytic cell function and inducing the release of cytokines (interleukin [IL] 1, IL-2, IL-6, IL-12, IL-18, tumor necrosis factor alpha, interferon gamma) and regulate the local inflammatory response by upregulating transforming growth factor beta and IL-10. ${ }^{34}$

In addition, Lactobacillus promotes mucin secretion, forms a mucus barrier, and blocks the invasion and adhesion of pathogenic bacteria. Moreover, probiotics synthesize short-chain fatty acids, reduce the intestinal $\mathrm{pH}$, and inhibit the colonization of acid-resistant pathogens, for example, Pseudomonas aeruginosa. ${ }^{10}$ Furthermore, high safety, low cost, and low toxicity are also advantages of probiotics. For VAP prevention, the specific probiotic strains, patient population, optimal dosage, and proper administration are still challenges that need to be considered in further research; however, our meta-analysis may provide some meaningful clues. Probiotics seem to reduce VAP incidence caused by $P$. aeruginosa, although this finding is tempered by the fact that $P$. aeruginosa is the most common Gram-negative pathogen associated with VAP. Moreover, Synbiotic 2000Forte may be an effective probiotic strain to prevent VAP.

There may be a certain amount of heterogeneity in our study. The strong influences were the differences in the trial 
Table 4. Subgroup Analysis for the Primary Outcome

\begin{tabular}{|c|c|c|c|}
\hline Outcome or Subgroup Title & Studies, no. & Subjects, $N$ & Effect Size \\
\hline Different species of probiotics & 13 & 1,875 & $0.62(0.45,0.85)$ \\
\hline $\begin{array}{l}\text { Lactobacillus rhamnosus } G G+\text { Lactobacillus casei }+ \\
\text { Lactobacillus acidophilus }+ \text { Bifidobacterium bifidum }\end{array}$ & 1 & 149 & $1.56(0.74,3.30)$ \\
\hline Lactobacillus casei rhamnosus & 2 & 340 & $0.62(0.21,1.81)$ \\
\hline Synbiotic 2000 Forte & 4 & 509 & $0.52(0.30,0.90)$ \\
\hline Lactobacillus plantarum 299 & 2 & 385 & $0.84(0.42,1.68)$ \\
\hline L. casei & 1 & 150 & $0.76(0.37,1.57)$ \\
\hline Bifidobacterium breve + L. casei & 1 & 72 & $0.18(0.06,0.55)$ \\
\hline $\begin{array}{l}\text { Bifidobacterium longum }+ \text { Lactobacillus bulgaricus }+ \\
\text { Streptococcus thermophilus }\end{array}$ & 1 & 35 & $0.36(0.09,1.43)$ \\
\hline Live Bacillus subtilis + Enterococcus faecalis & 1 & 235 & $0.56(0.33,0.95)$ \\
\hline Different diagnostic criteria for VAP & 13 & 1,875 & $0.62(0.45,0.85)$ \\
\hline Microbiologically confirmed diagnosis & 7 & 986 & $0.82(0.55,1.22)$ \\
\hline Clinical diagnosis & 4 & 704 & $0.57(0.40,0.80)$ \\
\hline Inexplicit diagnostic criteria & 2 & 185 & $0.22(0.10,0.50)$ \\
\hline Different trial design & 13 & 1,875 & $0.62(0.45,0.85)$ \\
\hline Double-blind studies & 6 & 885 & $0.72(0.44,1.19)$ \\
\hline Single-blind studies & 3 & 220 & $0.25(0.13,0.51)$ \\
\hline Open-label studies & 4 & 770 & $0.68(0.47,0.97)$ \\
\hline Different pathogens & 8 & 5,605 & $0.71(0.54,0.92)$ \\
\hline Acinetobacter baumannii & 7 & 989 & $0.73(0.42,1.25)$ \\
\hline Enterobacteriaceae & 7 & 1,119 & $0.97(0.54,1.76)$ \\
\hline Haemophilus influenzae & 2 & 397 & $0.33(0.05,2.14)$ \\
\hline Klebsiella pneumoniae & 4 & 345 & $0.81(0.24,2.66)$ \\
\hline Methicillin-resistant Staphylococcus aureus & 4 & 782 & $0.69(0.31,1.53)$ \\
\hline Pseudomonas aeruginosa & 8 & 1,191 & $0.54(0.32,0.91)$ \\
\hline Stenotrophomonas maltophilia & 4 & 782 & $0.70(0.17,2.97)$ \\
\hline
\end{tabular}



Fig. 12. A contour-enhanced meta-analysis funnel plot for ventilator-associated pneumonia incidence. 


\section{PROBIOTICS FOR VAP PREVENTION}

design and VAP diagnostic criteria. We performed subgroup analyses based on the differences in trial design and diagnostic criteria. However, the subgroup analysis of the double-blind studies showed no effect of probiotics compared with controls on the prevention of VAP, which meant that the role of probiotics may be inflated by studies with flawed designs. Likewise, no significant difference was found in the subgroup in which VAP was confirmed microbiologically. Different diagnostic criteria may affect the authenticity of the clinical results; hence, explicit and consistent diagnostic criteria are necessary in future studies. In addition, the analysis involved subjects with a variety of diseases, such as trauma, surgery, and sepsis, which may have led to different risks of VAP infection. The difference between probiotic strains, dosages, and delivery methods also reduced the comparability among the studies.

There were other limitations to our study. Due to the restriction of the retrieval strategy, we could not retrieve all documents that met the inclusion criteria. In addition, the methodological quality of the included studies was low, with several RCTs clearly lacking illustrations of randomness, allocation concealment, and blinding, among other factors, which increased difficulty in the risk of bias assessment. Moreover, our conversion of missing means and SDs may have led to inaccurate extrapolation values. Also, significant potential publication bias existed in the study.

\section{Conclusions}

An analysis of the available evidence demonstrated that probiotics can reduce the incidence of VAP. Further largescale, well-designed clinical trials are required to validate this effect and to determine the optimum probiotic strains for the prevention of VAP.

\section{REFERENCES}

1. Wolfensberger A, Meier MT, Clack L, Schreiber PW, Sax H. Preventing ventilator-associated pneumonia-a mixed-method study to find behavioral leverage for better protocol adherence. Infect Control Hosp Epidemiol 2018;39(10):1222-1229.

2. Nasiriani K, Torki F, Jarahzadeh MH, Rashidi Maybodi F. The effect of brushing with a soft toothbrush and distilled water on the incidence of ventilator-associated pneumonia in the intensive care unit. Tanaffos 2016;15(2):101-107.

3. Dudeck MA, Weiner LM, Allen-Bridson K, Malpiedi PJ, Peterson KD, Pollock DA, et al. National Healthcare Safety Network (NHSN) report, data summary for 2012, device-associated module. Am J Infect Control 2013;41(12):1148-1166.

4. Zeng J, Wang CT, Zhang FS, Qi F, Wang SF, Ma S, et al. Effect of probiotics on the incidence of ventilator-associated pneumonia in critically ill patients: a randomized controlled multicenter trial. Intensive Care Med 2016;42(6):1018-1028.

5. Meidani M, Khorvash F, Abbasi S, Cheshmavar M, Tavakoli H. Oropharyngeal irrigation to prevent ventilator-associated-pneumonia: comparing potassium permanganate with chlorhexidine. Int J Prev Med 2018;9:93.
6. Sharma G, Im SH. Probiotics as a potential immunomodulating pharmabiotics in allergic diseases: current status and future prospects. Allergy Asthma Immunol Res 2018;10(6):575-590.

7. Boulangé CL, Neves AL, Chilloux J, Nicholson JK, Dumas ME. Impact of the gut microbiota on inflammation, obesity, and metabolic disease. Genome Med 2016;8(1):42.

8. Meijnikman AS, Gerdes VE, Nieuwdorp M, Herrema H. evaluating causality of gut microbiota in obesity and diabetes in humans. Endocr Rev 2018;39(2):133-153.

9. Liu KX, Zhu YG, Zhang J, Tao LL, Lee JW, Wang XD, Qu JM. Probiotics' effects on the incidence of nosocomial pneumonia in critically ill patients: a systematic review and meta-analysis. Crit Care 2012;16(3):R109.

10. Karacaer F, Hamed I, Özogul F, Glew RH, Özcengiz D. The function of probiotics on the treatment of ventilator-associated pneumonia (VAP): facts and gaps. J Med Microbiol 2017;66(9):1275-1285.

11. Boyer AF, Kollef MH. Probiotics for ventilator-associated pneumonia: the need for a large, multicenter, randomized controlled trial. Chest 2013;143(3):590-592.

12. Giamarellos-Bourboulis EJ, Bengmark S, Kanellakopoulou K, Kotzampassi K. Pro- and synbiotics to control inflammation and infection in patients with multiple injuries. J Trauma 2009;67(4): 815-821.

13. Kotzampassi K, Giamarellos-Bourboulis EJ, Voudouris A, Kazamias P, Eleftheriadis E. Benefits of a synbiotic formula (Synbiotic 2000Forte) in critically Ill trauma patients: early results of a randomized controlled trial. World J Surg 2006;30(10):1848-1855.

14. Morrow LE, Kollef MH, Casale TB. Probiotic prophylaxis of ventilator-associated pneumonia: a blinded, randomized, controlled trial. Am J Respir Crit Care Med 2010;182(8):1058-1064.

15. Shimizu K, Yamada T, Ogura H, Mohri T, Kiguchi T, Fujimi S, et al. Synbiotics modulate gut microbiota and reduce enteritis and ventilator-associated pneumonia in patients with sepsis: a randomized controlled trial. Crit Care 2018;22(1):239.

16. Spindler-Vesel A, Bengmark S, Vovk I, Cerovic O, Kompan L. Synbiotics, prebiotics, glutamine, or peptide in early enteral nutrition: a randomized study in trauma patients. JPEN J Parenter Enteral Nutr 2007;31(2):119-126.

17. Klarin B, Adolfsson A, Torstensson A, Larsson A. Can probiotics be an alternative to chlorhexidine for oral care in the mechanically ventilated patient? A multicentre, prospective, randomised controlled open trial. Crit Care 2018;22(1):272.

18. Mahmoodpoor A, Hamishehkar H, Asghari R, Abri R, Shadvar K, Sanaie S. Effect of a probiotic preparation on ventilator-associated pneumonia in critically ill patients admitted to the intensive care unit: a prospective double-blind randomized controlled trial. Nutr Clin Pract 2019;34(1):156-162.

19. Luo D, Wan X, Liu J, Tong T. Optimally estimating the sample mean from the sample size, median, mid-range, and/or mid-quartile range. Stat Methods Med Res 2018;27(6):1785-1805.

20. Wan X, Wang W, Liu J, Tong T. Estimating the sample mean and standard deviation from the sample size, median, range and/or interquartile range. BMC Med Res Methodol 2014;14:135.

21. Higgins J, Green S. Cochrane handbook for systematic reviews of intervensions. 5.1.0. The Cochrane Collaboration. Available at: http:// handbook.cochrane.org. Accessed on January 8, 2020.

22. Barraud D, Blard C, Hein F, Marçon O, Cravoisy A, Nace L, et al. Probiotics in the critically ill patient: a double blind, randomized, placebo-controlled trial. Intensive Care Med 2010;36(9):1540-1547.

23. Forestier C, Guelon D, Cluytens V, Gillart T, Sirot J, De Champs C. Oral probiotic and prevention of Pseudomonas aeruginosa infections: a randomized, double-blind, placebo-controlled pilot study in intensive care unit patients. Crit Care 2008;12(3):R69. 


\section{PROBIOTICS FOR VAP PREVENTION}

24. Knight DJ, Gardiner D, Banks A, Snape SE, Weston VC, Bengmark S, Girling KJ. Effect of synbiotic therapy on the incidence of ventilator associated pneumonia in critically ill patients: a randomised, doubleblind, placebo-controlled trial. Intensive Care Med 2009;35(5):854861.

25. Oudhuis GJ, Bergmans DC, Dormans T, Zwaveling JH, Kessels A, Prins $\mathrm{MH}$, et al. Probiotics versus antibiotic decontamination of the digestive tract: infection and mortality. Intensive Care Med 2011;37 (1):110-117.

26. Weng H, Li JG, Mao Z, Feng Y, Wang CY, Ren XQ, Zeng XT. Probiotics for preventing ventilator-associated pneumonia in mechanically ventilated patients: a meta-analysis with trial sequential analysis. Front Pharmacol 2017;8:717.

27. Rongrungruang Y, Krajangwittaya D, Pholtawornkulchai K, Tiengrim S, Thamlikitkul V. Randomized controlled study of probiotics containing Lactobacillus casei (Shirota strain) for prevention of ventilatorassociated pneumonia. J Med Assoc Thai 2015;98(3):253-259.

28. Tan M, Zhu JC, Du J, Zhang LM, Yin HH. Effects of probiotics on serum levels of Th1/Th2 cytokine and clinical outcomes in severe traumatic brain-injured patients: a prospective randomized pilot study. Crit Care 2011;15(6):R290.
29. Bo L, Li J, Tao T, Bai Y, Ye X, Hotchkiss RS, et al. Probiotics for preventing ventilator-associated pneumonia. Cochrane Database of Syst Rev2014;(10):CD009066.

30. Siempos II, Ntaidou Tk, Falagas ME. Impact of the administration of probiotics on the incidence of ventilator-associated pneumonia: a meta-analysis of randomized controlled trials. Crit Care Med 2010;38 (3):954-962.

31. Banupriya B, Biswal N, Srinivasaraghavan R, Narayanan P, Mandal J. Probiotic prophylaxis to prevent ventilator associated pneumonia (VAP) in children on mechanical ventilation: an open-label randomized controlled trial. Intensive Care Med 2015;41(4):677-685.

32. Li XC, Wang JZ, Liu YH. Effect of probiotics on respiratory tract pathogen colonization in neonates undergoing mechanical ventilation [in Chinese with English abstract]. Zhongguo Dang Dai Er Ke Za Zhi 2012;14(6):406-408.

33. Wang J, Liu X, Ariani F, Tao LL, Zhang J, Qu JM. Probiotics for preventing ventilator-associated pneumonia: a systematic review and meta-analysis of high-quality randomized controlled trials. PloS One 2013;8(12):e83934.

34. Gill HS, Guarner F. Probiotics and human health: a clinical perspective. Postgrad Med J 2004;80(947):516-526. 